\title{
Desempeño financiero en las organizaciones: Análisis desde la gerencia general Chairman
}

\author{
Freire Quintero, César Enrique ${ }^{1}$ \\ Soto Tobar, Tania Alexandra ${ }^{2}$ \\ Mendieta Gonzabay, Daniela Nathaly ${ }^{3}$
}

\section{Resumen}

El propósito de este estudio fue investigar el efecto de la Dualidad del Gerente General (CEO) Chairman sobre el desempeño financiero de las empresas ecuatorianas. La investigación tiene una lógica deductiva, enfoque cuantitativo, diseño no experimental, alcance correlacional y fuentes de datos primaria y secundaria. Para la comprobación de la hipótesis general del modelo se aplicó estadísticas de regresión múltiple, ajuste de datos y test VIF para evaluar la multicolinealidad; a partir de una muestra de 343 CEO's con un nivel de confianza del 95\% y un margen de error del $5 \%$. Los hallazgos indicaron que la Dualidad CEO no está relacionada positivamente con el desempeño financiero (ROA), sin embargo, se acotó que otras variables como el endeudamiento resultó significativo para explicar el desempeño financiero en los modelos uno y dos y el personal resultó significativo para explicar el desempeño financiero en el modelo uno.

Palabras clave: Dualidad CEO Chairman; desempeño financiero; regresión.

Recibido: 20.03.19 Aceptado: 20.06.19

1 Doctorado (c) en Administración de Empresas, Mgs. Economía y Dirección de Empresas, Mgs. En finanzas y Proyectos. Docente en la Carrera de Negocios Internacionales, Facultad de Ciencias Económicas y Administrativas, Universidad Católica de Santiago de Guayaquil, Guayaquil, Ecuador, E-mail: cesar.freire@cu.ucsg.edu.ec

2 Estudiante de la Carrera de Administración de Empresas DUAL, Facultad de Ciencias Económicas y Administrativas, Universidad Católica de Santiago de Guayaquil, Guayaquil, Ecuador, E-mail: taniasototobar@gmail.com

3 Estudiante de la Carrera de Gestión Empresarial Internacional, Facultad de Ciencias Económicas y Administrativas, Universidad Católica de Santiago de Guayaquil, Guayaquil, Ecuador, E-mail: danielanmg@hotmail.com 


\title{
Financial performance in organizations: Analysis from the general management Chairman
}

\begin{abstract}
The purpose of this study was to investigate the effect of the Chief Executive Officer (CEO) Chairman Duality on the financial performance of Ecuadorian companies. The research has a deductive logic, quantitative approach, non-experimental design, correlational scope and primary and secondary data sources. For the verification of the general hypothesis of the model, multiple regression statistics, data adjustment and VIF test were applied to evaluate the multicollinearity; from a sample of 343 CEOs with a confidence level of $95 \%$ and a margin of error of $5 \%$. The findings indicated that CEO Duality is not positively related to financial performance (ROA), however, it was noted that other variables such as indebtedness were significant to explain the financial performance in models one and two and the staff was significant in explaining financial performance in model one.
\end{abstract}

Key words: Duality CEO Chairman, financial performance, Regression

\section{Introducción}

El propósito de este estudio es investigar el efecto de la Dualidad del Gerente General (CEO) Chairman sobre el desempeño financiero de las empresas ecuatorianas partiendo de que los miembros de la junta no mantienen niveles constantes de monitoreo. El desempeño se ve afectado por la dualidad del CEO incrementando la atención a los procesos de control. El Chairman dada la naturaleza de sus funciones hace que también se vea afectado el desempeño en la organización. Así mismo la presencia de la dualidad también reduce la asignación de atención de los consejos al monitoreo.

El problema radica que en la existencia de la dualidad CEO-Chairman se eleva el riesgo de una mala decisión, incentivando el problema principal agente y esto a su vez podría estar afectando al desempeño financiero de la organización. De esta forma se justifica teóricamente en el aporte al problema principal agente. Así mismo, la investigación en lo práctico aporta para el establecimiento de los buenos principios del gobierno corporativo; entonces, el estudio de estas variables en el ámbito metodológico parte en presentar varias opciones de modelos para analizar el ajuste de los datos ante variaciones matemáticas de estos.

\section{Bases teóricas de la dualidad CEO-Chairman}

CEO por sus siglas en inglés, proviene de la expresión Chief Executive Officer puede ser traducido como consejero delegado o director general (Pérez \& Merino, 2018). "Se trata del 
Desempeño financiero en las organizaciones: Análisis desde la gerencia general

Chairman

Freire Quintero, César Enrique; Soto Tobar, Tania Alexandra y Mendieta Gonzabay,

Daniela Nathaly

máximo cargo de responsabilidad dentro de la administración de la organización" (Garcia, s.f.). Kraft (2018) indicó que un CEO es un líder de tiempo completo y es el principal responsable de la toma de decisiones de la compañía. Martínez (2017) añadió que los CEO's al ser la máxima autoridad dentro de la empresa deben estar capacitados todo el tiempo para mejorar el desempeño financiero de la organización.

EI CEO es quien lidera la empresa y se encarga de establecer procesos estratégicos. En la realidad ecuatoriana, al CEO se lo puede definir como gerente general o representante legal de la compañía. Por lo tanto, al CEO se define como la persona que lidera administrativamente a la empresa, con capacidad legal para la toma de decisiones.

Las funciones que un CEO desarrolla son a) impulsar los proyectos; b) generar nuevos negocios; c) detectar y captar nuevas oportunidades de negocio; d) generar y promover una cultura organizativa; e) liderar y ser un mentor para el equipo de trabajo; f) dar seguimiento a los indicadores clave de rendimiento (Martínez, 2018). También Kraft (2018) señala que una de las funciones del CEO es hacer responsables a otros ejecutivos cuando las cosas no salen como deberían. De igual manera, García (s.f.) añadió que el CEO puede llegar a integrarse como parte de la junta directiva, incluso como presidente debido a que unas de sus funciones principales es actuar como puente de comunicación entre la empresa y la junta directiva.

El Chairman es el encargado de guiar al consejo directivo, la conceptualización del este es ser un líder de medio tiempo que administra los negocios y actividades de la junta y brinda orientación a otros miembros del directorio (Kraft, 2018). El Chairman es usualmente el dueño o el fundador de la compañía (Thattil, 2017) sin embargo, este puede ser externo. En síntesis, el Chairman se define como el presidente del directorio quien está encargado de dirigir las sesiones ordinarias y extraordinarias que se realicen como directorio.

Tanto el CEO como el Chairman, ambos son figuras con un alto nivel de poder dentro de la organización y tienen la potestad para la toma de decisiones. Por ende, un CEO tiene la responsabilidad de informar a la junta directiva, el Chairman funciona como un jefe informal del CEO. Sin embargo, en términos de realmente influir en una empresa, un CEO es en general mucho más poderoso (Gorne, 2017).

El tema de la dualidad CEOChairman ha sido controversial en el último siglo, muchas empresas se han planteado sobre si los roles CEOChairman deberían manejarse por separado. Este tema Tribbett (2012) lo manifestó en su investigación sobre la separación de la dualidad CEO Chairman, en donde cuestiona el funcionamiento de algunas empresas como JPMorgan Chase, Avon Products, entre otros y demuestra que en algunos casos ha sido el resultado de un debate rutinario por parte de la junta.

Afza, Yusof, Ishak y Aripin (2014) en su análisis sobre si las características entre CEO y Chairman influyen en el desempeño de las empresas vinculadas con el gobierno, exponen que un CEO que posee una alta formación académica aporta un invaluable capital humano para la empresa. Asimismo Lytkina, Rosenkoetter, Kincaid y Wang (2017) explican que los CEO's con un alto nivel de educación poseen la habilidad de 
tomar decisiones al contar apenas con el $65 \%$ total de la información, pues sienten la necesidad de tomar una decisión inmediata y no esperan a tener la información perfecta.

Los roles de CEO y Chairman tienen efectos importantes sobre el desempeño financiero de la empresa, puesto que ambos cumplen funciones similares que ayudan al crecimiento de la organización, en algunos casos las empresas tienen a una misma persona para cumplir el rol de CEO-Chairman (Crook, Harkin, Mare, \& Salvatore, 2018). A su vez, Yang y Zhao (2014) informan que las empresas que cuentan con un CEO-Chairman conjunto tienen una ventaja de rendimiento después de un cambio importante en las condiciones competitivas financieras del sector. Belkhir (2008) afirma que los CEO de las compañías conjuntas más pequeñas tienen menos probabilidades de ser también Chairman, si se da por descontada la idea de que la separación de los títulos de CEO-Chairman mejora el control de la junta, esto puede interpretarse como evidencia de la efectividad de las juntas más pequeñas.

Por otra parte, Pathan (2009) examina los efectos de la dualidad CEO-Chairman y encuentran que un CEO-Chairman tiene un efecto negativo en el riesgo bancario. Yang y Zhao (2014) alegan que la separación de estos dos roles tiene un efecto negativo en la toma de decisiones puesto que se vuelve confuso. Por el contrario, varios estudios empíricos llegan a la conclusión opuesta: que al no separar la dualidad de CEO-Chairman tienen efectos adversos en el desempeño de las empresas (Duru, lyengar, \& Zampelli, 2016). Brickley, Coles y Jarell (1997) también argumentaron que separar los roles de CEO-Chairman conlleva costos que superan las ventajas en el caso de las corporaciones no financieras en el caso de Estados Unidos.

Tener un solo jefe en la organización genera deficiencia en la dualidad de mando, así lo expresa Dumois (2016) y sostiene que solamente genera deficiencias de la dualidad de mando y falta de claridad organizacional. Aunque la dualidad de CEO-Chairman puede tener un impacto negativo en la organización existen otros motivos por los cuales un CEO puede fracasar. Stein y Capapé (2009) explican las causas del fracaso del director general o CEO en su estudio sobre las causas del fracaso de un CEO y en la mayoría de los casos el fracaso de los mismos depende de factores exógenos que ellos no pueden controlar.

Otro aspecto negativo en la dualidad de los mandos es la inoperancia de las empresas al no tener sus conceptos bien aplicados de liderazgo. "Cuando hay dualidad de mando, todos quieren meterse en todo aunque no sea de su área de competencia no hay claridad en la actividad y la organización complica su actividad causando diversos tipos de problemas" (Vides, 2010).

\section{Desempeño financiero en las organizaciones}

"El desempeño financiero es uno de los indicadores que se utilizan para medir el éxito de una institución en términos de su rentabilidad" (Mendiola, 2015). Al tratar un tema de importancia, en estos últimos 20 años, como lo es la dualidad CEO-Chairman pueden verse envueltos diversos aspectos negativos en cuanto a desempeño financiero; por ejemplo fuertes impactos en las medidas de rentabilidad contables (Palmon \& Wald, 2002). Por lo tanto, 
uno de los indicadores más importantes es el de rentabilidad financiera. De esta forma, Daza (2016) manifestó que "la rentabilidad puede considerarse, a nivel general, como la capacidad o aptitud de la empresa para generar un excedente, partiendo de un conjunto de inversiones efectuadas" (p.268). Nuevamente Argüelles et al. (2018) añadieron que los indicadores de rentabilidad son útiles para medir que tan efectiva es la administración de la empresa, controlando de esta manera el costo y el gasto y al mismo tiempo poder convertir las ventas en utilidades, reflejándolos con la siguiente ecuación:

Rentabilidad sobre Activos $($ ROA $)=\frac{\text { Utilidad } \mathrm{Neta}}{\text { TotaldelActivos }}$

(Ecuación 1)

Cuando una persona desarrolla el mismo rol, se ve más interesado por sus objetivos personales; dejando a un lado los de la organización incurriendo en gastos no necesarios y afectando la rentabilidad financiera de la compañía (Tuggle, Sirmon, Reutzel, \& Bierman, 2010). La elección de las estructuras de liderazgo del CEO y Chairman deben estar sujetas a políticas relacionadas con el desempeño financiero de la empresa con respecto a cualquier elemento fundamental de las estructuras organizacionales (Dalton \& Dalton, 2011). Las estructuras de gobierno corporativo no operan en un vacío, por lo tanto, los mecanismos de gobierno corporativo deben ser considerados y evaluados en conjunto; la relación entre las características de la junta y el desempeño financiero no es una relación monótona (Wahba, 2015).

\section{Impacto de la dualidad en el desempeño financiero}

Según la literatura existente implícitamente asume que la relación entre la composición de la junta o la estructura de liderazgo de la junta y el desempeño financiero es directa (Urban, 2019). La dualidad del CEO tiene impactos negativos estadísticamente significativos en el desempeño financiero de la empresa; este efecto es moderado positivamente por la independencia de la junta (Oh, Chang, \& Kim, 2018). La dualidad puede reducir el desempeño de la empresa a través de la consolidación de la gestión, no obstante, puede proporcionar beneficios a la firma en presencia de la vigilancia de la junta (Duru, lyengar, \& Zampelli, 2016).

Finalmente se puede concluir que la dualidad CEO-Chairman afecta altamente al desempeño financiero de la empresa ya sea por la falta de control o porque una sola persona toma las decisiones estratégicas sin pasar por un filtro como lo es la junta directiva. Si bien es cierto han existido fluctuaciones con el paso de los años; muchos autores señalan perspectivas diferentes lo que ha hecho que se conceptualice de manera equívoca los beneficios de una dualidad volviéndose, al final, un fenómeno complejo (Yasser \& Mamun, 2016). Por lo tanto, esto presume la siguiente hipótesis general de investigación:

\section{$H_{1}$ :La Dualidad CEO-Chairman} tiene una relación positiva con el desempeño financiero

El presente estudio emplea un modelo positivista de lógica deductiva con un enfoque de tipo cuantitativo, de diseño no experimental de tal manera que describe al sujeto de estudio en su 
contexto natural, sin intervención del investigador (Hernández, Fernández, \& Baptista, 2010). La investigación presenta un alcance correlacional debido al análisis de la relación entre la dualidad del CEO Chairman y su relación con el desempeño financiero de la organización. Las fuentes de información empleadas son primaria debido a que el estudio se recopiló a través del instrumento de recolección de datos; es decir, por medio de cuestionarios debidamente validados por sus respectivos autores, obteniendo de esta forma la variable Dualidad CEO Chairman como independiente del estudio; para la cuantificación de la variable se utiliza un cuestionario de pregunta dicotómica de dos alternativas Sí y No. También se considera fuentes secundarias donde se extrajo valores financieros de la Superintendencia de Compañías (Supercias) en la cual se obtiene la variable Desempeño Financiero (ROA) como dependiente en la investigación.

Las técnicas estadísticas empleadas son de regresión múltiple y para la comprobación del ajuste de datos se utiliza los análisis de multicolinealidad en la cual se aplica el Factor de Inflación de la Varianza (VIF). Se emplea un muestreo no probabilístico, estratificado por sectores y dualidad del CEO Chairman con un nivel de muestra de 343 CEO's con un nivel de confianza del $95 \%$ y un margen de error del $5 \%$. En conclusión, los métodos estadísticos utilizados sirvieron para poder responder la principal pregunta de investigación.

Para el levantamiento de información se encuestó a 343 gerentes de organizaciones privadas formalmente constituidas en el Ecuador para conocer si el CEO cumplía con las mismas funciones que el Chairman. Adicionalmente para capturar las variables desempeño financiero, endeudamiento sobre activo, personal, tipo de sector y si la empresa es familiar o no, se obtuvo a partir de fuentes secundarias de la Superintendencia de Compañía, Valores y Seguros (Supercias). El análisis de los datos se realizó por medio el programa estadístico Stata. A continuación, se describe cada uno de los modelos de regresión múltiple:

$$
\begin{aligned}
& \text { Modelo 1: } R \boldsymbol{O A}=\beta_{0}+\beta_{1}(\text { DUALIDADDN })+\beta_{2}(\text { ENDACT })+\beta_{3}(\text { PERSONAL })+ \\
& \beta_{4}(S S)+\beta_{5}(S P)+\beta_{5}(F A M) \text {. }
\end{aligned}
$$

(Ecuación 2)

$$
\begin{aligned}
& \text { Modelo 2: ROA }=\beta_{0}+\beta_{1}(\text { DUALIDADDN })+\beta_{2}(\ln E N D A C T)+\beta_{3}(\ln P E R S O N A L)+ \\
& \beta_{4}(S S)+\beta_{5}(S P)+\beta_{5}(F A M)
\end{aligned}
$$

(Ecuación 3)

$$
\begin{aligned}
& \text { Modelo 3: } R O A I N=\beta_{0}+\beta_{1}(\text { DUALIDADDN })+\beta_{2}(\ln E N D A C T)+\beta_{3}(\ln \text { PERSONAL })+ \\
& \beta_{4}(S S)+\beta_{5}(S P)+\beta_{5}(F A M)
\end{aligned}
$$

(Ecuación 4) 
Desempeño financiero en las organizaciones: Análisis desde la gerencia general Chairman

Freire Quintero, César Enrique; Soto Tobar, Tania Alexandra y Mendieta Gonzabay, Daniela Nathaly

En el modelo 1 se presenta las variables sin ningún tipo de cambio, donde se aplica que el ROA es igual a la constante más la dualidad CEO Chairman másel endeudamiento sobre activos (ENDACT), personal y las variables Dummies del sector secundario (SS), primario (SP) y empresas familiares (FAM). Para el modelo 2 se plantea las variables con transformaciones, donde se aplica logaritmo natural al endeudamiento sobre activos (InENDACT) y al personal
(InPERSONAL). Finalmente, en el modelo 3 se plantea la inversa del ROA (ROAIN).

Los resultados obtenidos en este estudio, se recogen en esta sección, la cual el análisis parte para cada modelo planteado y posteriormente corroborar las hipótesis planteadas en esta investigación. La Tabla 1, 2 y 3 muestran diversos submodelos $\mathrm{m} 1, \mathrm{~m} 2$, m3 m4 y M5 donde se puede observar el dinamismo de los coeficientes al ingresar una variable más en los mismos

$$
\begin{aligned}
& \text { Modelo 1: } \boldsymbol{R} \boldsymbol{O A}=\boldsymbol{\beta}_{0}+\boldsymbol{\beta}_{1}(\text { DUALIDADDN })+\beta_{2}(\text { ENDACT })+\beta_{3}(\text { PERSONAL })+ \\
& \beta_{4}(S S)+\beta_{5}(S P)+\beta_{5}(F A M) \text {. }
\end{aligned}
$$

\section{Tabla 1}

\begin{tabular}{|c|c|c|c|c|c|}
\hline Variable & m1 & m2 & m3 & m4 & m5 \\
\hline \multirow[t]{3}{*}{ DUALIDADN } & $-1.45^{a}$ & -1.60 & -1.62 & -1.59 & -1.50 \\
\hline & $1.38^{\mathrm{b}}$ & 1.33 & 1.34 & 1.33 & 1.32 \\
\hline & $0.29^{c}$ & 0.23 & 0.23 & 0.23 & 0.26 \\
\hline \multirow[t]{3}{*}{ ENDACT } & & 31.84 & 31.83 & 31.60 & 31.62 \\
\hline & & 12.55 & 12.55 & 12.44 & 12.45 \\
\hline & & 0.01 & 0.01 & 0.01 & 0.01 \\
\hline \multirow[t]{3}{*}{ PERSONAL } & & & -0.00 & -0.00 & -0.00 \\
\hline & & & 0.00 & 0.00 & 0.00 \\
\hline & & & 0.31 & 0.02 & 0.02 \\
\hline \multirow[t]{3}{*}{ SPRIMARIO } & & & & 2.78 & 2.76 \\
\hline & & & & 1.88 & 1.88 \\
\hline & & & & 0.14 & 0.14 \\
\hline \multirow[t]{3}{*}{ SSECUNDARIO } & & & & 1.03 & 1.01 \\
\hline & & & & 1.26 & 1.27 \\
\hline & & & & 0.42 & 0.43 \\
\hline \multirow[t]{2}{*}{ FAM } & & & & & -0.16 \\
\hline & & & & & 1.04 \\
\hline
\end{tabular}

\section{Estadísticos de regresión múltiple del Modelo 1}


pp. $890-903$

Revista Venezolana de Gerencia, Año 24 N$^{\circ} 87,2019$

\section{Cont... Tabla 1}

\begin{tabular}{rrrrrr}
\hline & & & & & 0.88 \\
CONSTANTE & 1.47 & -17.81 & -17.48 & -17.78 & -17.68 \\
& 1.38 & 7.07 & 6.95 & 7.06 & 7.02 \\
& 0.29 & 0.01 & 0.01 & 0.01 & 0.01 \\
\hline
\end{tabular}

a Se refiere a los coeficientes del modelo, ${ }^{b}$ representa los errores estándar del modelo, ${ }^{c}$ muestra el valor $p$ del coeficiente.

Fuente: elaboración propia

La Tabla 1 explica que con un nivel de significancia del $5 \%$, la variable Dualidad CEO Chairman no explica el Desempeño Financiero medido por el ROA. Por otra parte, ENDACT es significativa al $1 \%$ con el ROA para todos los 5 submodelos.
En el submodelo 5 se puede observar que al $5 \%$ de significancia la variable endeudamiento sobre activos y personal resultan significativas. Por otro lado, las variables Dualidad, sector primario, sector secundario y empresa familiar no son significativas para el modelo.

Modelo 2: $\quad R O A=\beta_{0}+\beta_{1}($ DUALIDADDN $)+\beta_{2}(\ln E N D A C T)+\beta_{3}(\ln P E R S O N A L)+$ $\beta_{4}(S S)+\beta_{5}(S P)+\beta_{5}(F A M)$.

\section{Tabla 2}

Estadísticos de regresión múltiple del Modelo 2

\begin{tabular}{|c|c|c|c|c|c|}
\hline Variable & m1 & m2 & m3 & $\mathrm{m} 4$ & m5 \\
\hline \multirow[t]{3}{*}{ DUALIDADN } & $-1.45^{a}$ & -1.61 & -1.63 & -1.45 & -1.50 \\
\hline & $1.38^{\mathrm{b}}$ & 1.54 & 1.56 & 1.40 & 1.45 \\
\hline & $0.29^{c}$ & 0.30 & 0.30 & 0.30 & 0.30 \\
\hline \multirow[t]{3}{*}{ LN(ENDACT) } & & 1.92 & 1.92 & 1.71 & 1.69 \\
\hline & & 1.91 & 1.91 & 1.69 & 1.66 \\
\hline & & 0.31 & 0.31 & 0.31 & 0.31 \\
\hline \multirow[t]{3}{*}{ LN(PERSONAL) } & & & -0.15 & -0.44 & -0.51 \\
\hline & & & 0.17 & 0.44 & 0.51 \\
\hline & & & 0.39 & 0.32 & 0.32 \\
\hline \multirow[t]{3}{*}{ SPRIMARIO } & & & & 8.14 & 8.24 \\
\hline & & & & 7.79 & 7.87 \\
\hline & & & & 0.30 & 0.30 \\
\hline SSECUNDARIO & & & & 0.31 & 0.57 \\
\hline
\end{tabular}


Desempeño financiero en las organizaciones: Análisis desde la gerencia general Chairman

Freire Quintero, César Enrique; Soto Tobar, Tania Alexandra y Mendieta Gonzabay, Daniela Nathaly

\section{Cont... Tabla 2}

\begin{tabular}{|c|c|c|c|c|c|}
\hline & & & & 0.45 & 0.67 \\
\hline & & & & 0.49 & 0.40 \\
\hline FAM & & & & & -2.17 \\
\hline & & & & & 2.20 \\
\hline & & & & & 0.33 \\
\hline CONSTANTE & 1.47 & 3.15 & 3.70 & 3.45 & 4.78 \\
\hline & 1.38 & 3.01 & 3.57 & 3.30 & 4.63 \\
\hline & 0.29 & 0.30 & 0.30 & 0.30 & 0.30 \\
\hline
\end{tabular}

${ }^{a}$ Se refiere a los coeficientes del modelo, ${ }^{b}$ representa los errores estándar del modelo, ${ }^{c}$ muestra el valor $p$ del coeficiente.

Fuente: elaboración propia

En el modelo general 2 se aplicó logaritmo en las variables paramétricas de ENACT y Personal con el propósito de buscar un mejor ajuste al modelo y a la relación de las variables estudiadas, sin embargo, ninguna variable logró ser significativa para ningún submodelo.

Modelo 3: $\quad R O A I N=\beta_{0}+\beta_{1}($ DUALIDADDN $)+\beta_{2}(\ln E N D A C T)+\beta_{3}(\ln P E R S O N A L)+$ $\beta_{4}(S S)+\beta_{5}(S P)+\beta_{5}(F A M)$

\section{Tabla 3}

\section{Estadísticos de regresión múltiple del Modelo 3}

\begin{tabular}{|c|c|c|c|c|c|}
\hline Variable & $\mathrm{m} 1$ & $\mathrm{~m} 2$ & m3 & $\mathrm{m} 4$ & m5 \\
\hline \multirow[t]{3}{*}{ DUALIDADN } & $2,051.65^{a}$ & $2,080.47$ & $2,091.19$ & $2,047.60$ & $1,959.96$ \\
\hline & $1,978.44^{b}$ & $1,977.68$ & $1,997.29$ & $1,973.30$ & $1,892.88$ \\
\hline & $0.30^{c}$ & 0.29 & 0.30 & 0.30 & 0.30 \\
\hline \multirow[t]{3}{*}{ LN(ENDACT) } & & -353.38 & -354.22 & -415.98 & -456.37 \\
\hline & & 140.00 & 139.72 & 130.18 & 166.20 \\
\hline & & 0.01 & 0.01 & 0.00 & 0.01 \\
\hline \multirow[t]{3}{*}{ LN(PERSONAL) } & & & 64.86 & -169.85 & -261.95 \\
\hline & & & 121.39 & 138.40 & 226.75 \\
\hline & & & 0.59 & 0.22 & 0.25 \\
\hline \multirow[t]{3}{*}{ SPRIMARIO } & & & & $2,704.76$ & $2,842.97$ \\
\hline & & & & $2,604.03$ & $2,752.51$ \\
\hline & & & & 0.30 & 0.30 \\
\hline SSECUNDARIO & & & & $2,822.24$ & $3,172.28$ \\
\hline
\end{tabular}




\section{Cont... Tabla 3}

\begin{tabular}{|c|c|c|c|c|c|}
\hline & & & & $2,455.71$ & $2,821.34$ \\
\hline & & & & 0.25 & 0.26 \\
\hline \multirow[t]{3}{*}{ FAM } & & & & & $-2,875.04$ \\
\hline & & & & & 2,995.69 \\
\hline & & & & & 0.34 \\
\hline \multirow[t]{3}{*}{ CONSTANTE } & $-1,774.89$ & $-2,084.61$ & $-2,328.79$ & $-2,541.93$ & -776.06 \\
\hline & $1,975.77$ & $1,951.69$ & $2,348.60$ & $2,542.30$ & 781.71 \\
\hline & 0.37 & 0.29 & 0.32 & 0.32 & 0.32 \\
\hline
\end{tabular}

El modelo 3 empleó la inversa del ROA con el objetivo de lograr un mejor ajuste, sin embargo, en la Tabla 3 se observa que a un 0.05 nivel de significancia las variables expresas en los modelos mencionados no explican el desempeño financiero de las organizaciones. En el submodelo 5 se puede observar que la variable endeudamiento resulta significativa a un nivel de confianza del $95 \%$.

Para el desarrollo de los modelos propuestos se aplicó la prueba VIF donde los valores menores a 10 explica que tienen grados de multicolinealidad preocupante, de esta forma en los modelos generales se obtiene valores no superiores a 1.16 de tal forma que no existe problemas de multicolinealidad entre las variables. Además, mediante el análisis de los tres modelos generales de regresión múltiple se tiene que las variables independientes de Dualidad CEO Chairman y personal no son significativas para el ROA, por otro lado, la variable ENDACT tiene significancia para el desempeño financiero en el modelo 1 y 2 . Por lo tanto, se tiene evidencia estadística contundente de rechazar la hipótesis general del estudio, en la que se indica que La Dualidad CEO Chairman está relacionado de manera positiva con el Desempeño Financiero y justamente el aporte del conocimiento a partir de los resultados radica en no evidenciar a la dualidad CEO Chairman como un factor que puede afectar la variable dependiente de estudio.

Ciertos estudios afirman los resultados obtenidos dentro de esta investigación, Narwal y Jindal (2015) determinaron que los directores no ejecutivos y las reuniones del consejo influyen positivamente en la rentabilidad, pero no existe una relación significativa con la misma. De la misma forma, Arora y Sharma (2016) explicaron que existe una relación negativa entre la independencia del consejo y el desempeño de la empresa, por lo tanto la dualidad del CEO no se encuentra relacionada con ninguna medida de desempeño; de esta manera no parece ser un determinante crucial del desempeño de la empresa (Rashid, 2013).

Por otra parte, otras investigaciones afirman lo contrario a los análisis de estudio, Rajput (2015) concluyó que hay 
un impacto negativo y significativo de las reuniones del consejo y la presencia de directores no ejecutivos que pueden no tener compromiso total con la causa de la empresa debido a otros compromisos que limitan su contribución y beneficios financieros. Además, la estructura de liderazgo de una empresa o una doble posición ocupada por el CEO tiene una fuerte asociación positiva con el desempeño de la empresa (Muhammad \& Rehman, 2016). Esto significa que una sola persona que actúa como CEO y como Presidente de la junta influye en gran medida en la utilización óptima de los activos y el capital de la empresa, junto con varios otros factores (Bansal \& Sharma, 2016). Habib (2016); Latif y Shahid (2013) añadieron que la Dualidad CEO Chairman tiene correlaciones positivas significativas con el desempeño financiero de la organización ROA.

Narwal y Jindal (2015) acotaron que la no significancia entre las variables de estudio se ve influenciada por los grandes directorios lo cuales están asociados con una mayor profundidad de conocimiento intelectual, que a su vez ayuda a mejorar la toma de decisiones y el desempeño financiero. Arora y Sharma (2016) añadieron que si las empresas aplican los principios de una administración enfocada en el crecimiento laboral, entonces, las organizaciones logran los objetivos beneficiando el rendimiento de las mismas. Rashid (2013) examinó que no existe significancia entre la Dualidad CEO y la rentabilidad, mientras que una variable que influencia el desempeño financiero de las empresas es el salario de los CEO y colaboradores.

\section{Conclusiones}

En la realidad ecuatoriana, las acciones que ejecuta el CEO y el Chairman son monitoreadas constantemente por organismos públicos como la Supercia, lo cual provoca que se minimice el problema principal agente. Cuando hay control por parte de agentes externos a una organización puede ser posible que no apliquen las normativas de buen gobierno corporativo que castigan la presencia de la Dualidad CEO Chairman, en consecuencia en un mundo globalizado es relevante considerar todos los escenarios posibles a nivel empresarial.

La globalización y la privatización en el desarrollo de las economías ha sido un importante pilar para desenvolverse en las direcciones organizacionales enfrentando las competencias locales e internacionales, de tal forma que este estudio tuvo la importancia de evaluar el impacto de la dualidad CEO en el desempeño financiero. Por ende, se recomienda que para futuras investigaciones se añadan en el modelo las variables como el salario, el desarrollo laboral de los colaboradores y el intelecto de los directivos evaluando su impacto en el desempeño financiero.

\section{Referencias bibliográficas}

Afza, Noor, Yusof, MMohd, Ishak, Rokiah, \& Aripin, Norhani (8 de January de 2014), Do Characteristics of CEO and Chairman Influence GovernmentLinked Companies performance? Procedia - Social and Behavioral Sciences, No.109, pp 799-803.

Argüelles, Luis, Quijano, Román, Fajardo, Mario, Medina, Fernando, \& Cruz, Carlos (2018), El Endeudamiento como Indicador de Rentabilidad Financiera en las MIPYMES Turísticas de Campeche. Global Conference on Business and Finance Proceedings, vol. XIII No.1, pp 278-284. 
Arora, Alisson, \& Sharma, Carla (2016), Corporate governance and firm performance in developing countries: Evidence from India. Corporate Governance, vol. 16 No.2, pp 420-436.

Bansal, Norma, \& Sharma, Andy (2016), Audit committee, corporate governance and firm performance: Empirical evidence from India. International Journal of Economics and Finance, vol. 8 No.3, pp 1-31.

Belkhir, Mohamed (July de 2008), Board of Directors Size and Performance in the Banking Industry. International Journal of Managerial Finance.

Brickley, James, Coles, Joseph, \& Jarell, Garreth (1997), Leadership structure: Separating the CEO and Chairman of the Board. Journal of Corporate Finance, vol. 3 No.3, pp 189-220.

Crook, Harkin, Jonathan, Mare, Sean, \& Salvatore, Davide. (2018), Confusion Between the CEO and Chairman: Empirical. Obtenido de https://dx.doi. org/10.2139/ssrn.3317981

Dalton, Dan, \& Dalton, Catherine (11 de June de 2011), Integration of Micro and MacroStudiesin Governance Research: CEO Duality, Board Composition, and Financial Performance. Journal of Management, vol. 37 No.2, pp 404411.

Daza Izquierdo, Julio (2016), Crecimiento y rentabilidad empresarial en el sectorindustrial brasileño. Contaduría y Administración, vol. LXI No.2, pp 265-282.

Dumois, Carlos. (2016), La dualidad de mando en la práctica. El siglo.

Duru, Agustine, lyengar, Raghavan, \& Zampelli, Emily (2016), The dynamic relationship between CEO duality and firm performance: The moderating role of board independence. Journal of Business Research,vol. 69 No.10, pp

\section{9-4277.}

Garcia, Jose (s.f.), Economía simple.net. Obtenido de Definición de CEO: https:// www.economiasimple.net/glosario/ceo

Gorne, Martin (2 de October de 2017), Quora. Obtenido de Who is more powerful between the CEO and the chairman?: https://www.quora.com/ Who-is-more-powerful-between-theCEO-and-the-chairman

Habib, Mario (2016), Relationship between corporate governance and firm performance: A case study in Bangladesh. South African Journal of Business Management, vol. 46 No.4, pp 43-52.

Hernández, Renato, Fernández, Camila, \& Baptista, Paula. (2010), Metodología de la investigación. México: McGraw Hill.

Kraft, Debra (18 de June de 2018), Chron. Obtenido de CEO vs. Chairman: https://work.chron.com/ceo-vschairman-29866.html

Latif, Beshar, \& Shahid, Marianna (2013), Impact of corporate governance on firm performance: Evidence from sugar mills of Pakistan. European Journal of Business and Management, vol. 5 No.1, pp 51-59.

Lytkina, Elena, Rosenkoetter, Kim, Kincaid, Stephen, \& Wang, Dina(June de 2017), What sets successful CEOs apart. Harvard Business Review.

Martínez, Ignacio (5 de septiembre de 2017), Liderazgo, coaching y estrategias . Obtenido de Qué significa CEO y cuáles son sus principales funciones: http://ignaciomartineza.com/ liderazgo/que-significa-ceo/

Martínez, Maribel (8 de febrero de 2018), Expertos Negocios Online. Obtenido de Que es un CEO y las funciones del CEO en una empresa: https://www. expertosnegociosonline.com/que-es- 
Desempeño financiero en las organizaciones: Análisis desde la gerencia general

Chairman

Freire Quintero, César Enrique; Soto Tobar, Tania Alexandra y Mendieta Gonzabay,

Daniela Nathaly

un-ceo-significado-funciones/\#dos

Mendiola, Ana (2015), Portal FinDev. Obtenido de Desempeño financiero: https://www.findevgateway.org/es/ temas/desempe\%C3\%B1o-financiero

Muhammad, Hommuh, \& Rehman, Ammhed. U. (2016), The effect of corporate governance practices on firm performance: Evidence from Pakistan. The East Asian Journal of Business Management, vol. 6 No.1, pp 5-12.

Narwal, Kendra, \& Jindal, Susan (2015), The Impact of Corporate Governance on the Profitability: An Empirical Study of Indian Textile Industry. International Journal of Research in Management, vol.3 No.2, pp 81-85.

Oh, Won-Young, Chang, Young Kyun, \& Kim, Tae.-Yeol (1 de September de 2018), Complementary or Substitutive Effects? Corporate Governance Mechanisms and Corporate Social Responsibility. Journal of Management, vol. 44 No.7, pp 27162739.

Palmon, Oded, \& Wald, John (17 de April de 2002), Are two heads better than one? The impact of changes in management structure on performance by firm size. Journal of Corporate Finance, vol.8 No.3, pp 213-226.

Pathan, Sampiere (2009), Strong boards, CEO power and bank risk-taking. Journal of Banking and Finance, No.40, pp. 1340-1350. doi:https://doi. org/10.1016/j.jbankfin.2009.02.001

Pérez, Julian, \& Merino, María (2018), Definicion.de. Obtenido de Definicion de CEO: https://definicion.de/ceo/

Rajput, Nahjir (2015), Shareholder Types, Corporate Governance and Firm Performance: An Anecdote from Indian Corporate Sector. Asian Journal of Finance \& Accounting, vol. 7 No.1, pp 1-45.
Rashid, Ali (2013), Corporate governance, executive pay and firm performance: Evidence from Bangladesh. International Jornual of Management, vol. 30 No.2, pp 556570.

Stein, Guido, \& Capapé, Javier (2009), Causas del fracaso de un CEO: mapa de un debate. Revista empresa y humanismo, vol. 13 No.2, pp 183-236.

Thattil, Sascha (1 de October de 2017), Quora. Obtenido de Who is more powerful between the CEO and the chairman?: https://www.quora.com/ Who-is-more-powerful-between-theCEO-and-the-chairman

Tribbett, Charles (December de 2012), Splitting The CEO and Chairman Roles. The leading journal of corporate governance, vol.33 No.197.

Tuggle, Christopher, Sirmon, David, Reutzel, Christopher, \& Bierman, Leonard (06 de July de 2010), Commanding board of director attention: investigating how organizational performance and CEO duality affect board members' attention to monitoring. Strategic Management Journal, vol.31 No.9, pp 946-968.

Urban, Daniel (March de 2019), The effects of culture on CEO power: Evidence from executive turnover. Journal of Banking \& Finance, No.104, pp 5069.

Vides, Luis (13 de Octubre de 2010), Dualidad de Mando. Obtenido de http:// dualidaddemando.blogspot.com/

Wahba, Hayam (2015), The joint effect of board characteristics on financial performance: Empirical evidence from Egypt. Review of Accounting and Finance, vol.14 No.1, pp 20-40.

Yang, Tann, \& Zhao, Seah (2014), CEO duality and firm performance: Evidence from an exogenous shock to the competitive environment. Journal 
pp. $890-903$

Revista Venezolana de Gerencia, Año 24 N 87, 2019

of Banking and Finance, No.49, pp 534-552. doi:https://doi.org/10.1016/j. jbankfin.2014.04.008

Yasser, Qaiser Rafique, \& Mamun, Abdullah Al (15 de October de 2016), The relationship between board leadership structure and earnings management in Asia-Pacific. Accounting Research Journal, vol.29 No.4, pp 413-428.

- $\quad$ Esta obra está bajo una licencia de Creative Commons Reconocimiento-NoComercial- Compartirlgual 3.0 Unported. http://creativecommons.org/licenses/by-nc-sa/3.0/deed.es_ES 